\title{
Performing the unexplainable: Implicit task performance reveals individually reliable sequence learning without explicit knowledge
}

\author{
Daniel J. Sanchez, Eric W. Gobel, and Paul J. Reber \\ Northwestern University, Evanston, Illinois
}

\begin{abstract}
Memory-impaired patients express intact implicit perceptual-motor sequence learning, but it has been difficult to obtain a similarly clear dissociation in healthy participants. When explicit memory is intact, participants acquire some explicit knowledge and performance improvements from implicit learning may be subtle. Therefore, it is difficult to determine whether performance exceeds what could be expected on the basis of the concomitant explicit knowledge. Using a challenging new sequence-learning task, robust implicit learning was found in healthy participants with virtually no associated explicit knowledge. Participants trained on a repeating sequence that was selected randomly from a set of five. On a performance test of all five sequences, performance was best on the trained sequence, and two-thirds of the participants exhibited individually reliable improvement (by chi-square analysis). Participants could not reliably indicate which sequence had been trained by either recognition or recall. Only by expressing their knowledge via performance were participants able to indicate which sequence they had learned.
\end{abstract}

Experts in skilled motor performance are acutely aware of the distinction between the ability to perform a task and the ability to describe the operations involved in its execution. This dissociation leads to such effects as verbal overshadowing (Flegal \& Anderson, 2008; following Schooler \& Engstler-Schooler, 1990), in which consciously reflecting on motor movements hinders performance. Knowledge supporting skilled performance may sometimes be reportable verbally, but when conscious reflection produces interference, it implies that there may be separate representations for performance and reporting that can be put into competition.

Memory systems theory has provided a sizeable volume of research on the separate brain systems supporting these different memory types. Explicit, declarative memory supports verbal description and depends on the medial-temporal lobe (MTL) memory system, whereas implicit learning is supported by nondeclarative memory systems that improve performance (Milner, Squire, \& Kandel, 1998; Squire \& Knowlton, 2000). The basis for this research is the observation of preserved implicit memory in amnesic patients with MTL damage. When trying to learn new facts and events, these patients are typically severely impaired. However, certain forms of skill learning remain intact-for example, mirror tracing, mirrored text reading, and perceptual-motor sequence learning (Brooks \& Baddeley, 1976; Cohen \& Squire, 1980; Gabrieli, Corkin, Mickel, \& Growdon, 1993; Nissen \& Bullemer, 1987; Reber \& Squire, 1994).

Despite the abundance of neuropsychological evidence for multiple memory systems, clear dissociations between knowing "how" and reporting "what" have been difficult to observe in healthy participants. Functional neuroimaging with healthy participants has shown a dissociation between memory systems in some tasks (Reber, Gitelman, Parrish, \& Mesulam, 2003) but not in others (Willingham, Salidis, \& Gabrieli, 2002). Attempts to show behavioral dissociations often run into two types of problems: First, healthy participants have intact declarative memory capabilities and are able to remember events that occurred during learning, creating difficulties in separating explicit memory from knowledge of the skill being acquired (Willingham, Greeley, \& Bardone, 1993). Both types of memory often develop in parallel (Willingham \& Goedert-Eschmann, 1999), and the amount of explicit knowledge can range from minimal to extensive enough that participants can describe and cognitively manipulate their newly obtained memories (Smyth \& Shanks, 2008; Wilkinson \& Shanks, 2004).

Second, most studies of implicit skill learning find small learning effects that are reliable across groups of participants, making it difficult to be certain that individuals with poor explicit memory have significant implicit knowledge. The challenge is to develop a learning paradigm capable of producing robust implicit learning in individuals without concomitant explicit awareness. Since unforeseen methodological issues arise with completely novel implicit tasks (Chambaron, Ginhac, Ferrel-Chapus, $\&$ Perruchet, 2006), the learning paradigm reported here extends upon the extensive research history behind the serial reaction time (SRT) task. 
The new task reported here uses serial interception responses in a video-game-style display (serial interception sequence learning [SISL]). In a manner that is conceptually similar to the SRT task, participants are covertly trained on an embedded sequence of motor responses and show sequence-specific learning via improved performance on the trained sequence compared with on novel sequences. However, the SISL task requires responses to be made at a much more rapid pace and is challenging enough that performance is measured as the percentage of correct responses. The demand characteristics of the SISL task produce implicit knowledge in the form of a sequence-specific performance increase, while making explicit knowledge difficult to obtain (i.e., unexplainable).

\section{METHOD}

\section{Participants}

Thirty undergraduates (17 female, 13 male, mean age $=$ 18.7 years) from Northwestern University received course credit for participating.

\section{Materials}

The SISL task. Participants were seated at a computer and observed cues (blue circles), which scrolled vertically up the computer monitor from one of four horizontal locations toward four corresponding yellow target rings located near the top of the screen (Figure 1). Participants were instructed to press one of four associated keys when a circle overlapped with its target ring. A correct response was scored if the participant pressed the correct key when the corresponding cue was closer to the target zone than any other cue. Incorrect keypresses, multiple keypresses, and missed responses were scored as errors. Target rings flashed green in response to a keypress, but gave no feedback as to whether the response was correct. Cues moved with a velocity of $13 \%$ sec to reach the target zones $1,700 \mathrm{msec}$ after first appearing at the bottom of the screen. Multiple cues moved on the screen simultaneously so that subsequent motor responses could be planned.

Participants were not informed that the cues generally followed a repeating 12-item second-order conditional (SOC; Reed \& Johnson, 1994) sequence. In an SOC sequence, all single responses occur equally frequently and each possible nonrepetition pair of responses occurs exactly once (e.g., F-J-F-K-D-J-D-K-J-K-F-D). The next response cannot be predicted by frequency or by simple (first-order) association, but the next element can be predicted perfectly from the two preceding responses. During training, participants were assigned randomly to learn one of five possible SOC sequences. The other four sequences were used as foils for implicit and explicit posttests.

\section{Procedure}

The first phase of the experiment consisted of 48 blocks, each comprising 60 trials (2,880 trials total), with self-terminated breaks after every 8 blocks. The training was completed in roughly $35 \mathrm{~min}$. Each block included four repetitions of the participant's trained sequence and one novel SOC sequence ( $20 \%$ pseudorandom noise). To keep the motor responses from becoming monotonous, every sequence iteration (trained, noise, or foil) contained the same embedded 12-item timing sequence consisting of an equal number of short and long interstimulus intervals (ISIs; $\mathrm{L}-\mathrm{S}-\mathrm{L}-\mathrm{L}-\mathrm{S}-\mathrm{S}-\mathrm{L}-\mathrm{S}-\mathrm{L}-\mathrm{L}-\mathrm{S}-\mathrm{S}$ ). The ISIs were 350 and $700 \mathrm{msec}$ initially, but were decreased during training by 25 and $50 \mathrm{msec}$, respectively, after every 480 trials until they were a constant 225 and $550 \mathrm{msec}$, respectively, for both the implicit and recognition tests.

After training, three tests of sequence knowledge were administered. First, participants performed a 1,200-trial implicit test in which all five 12-item SOC sequences were presented four times

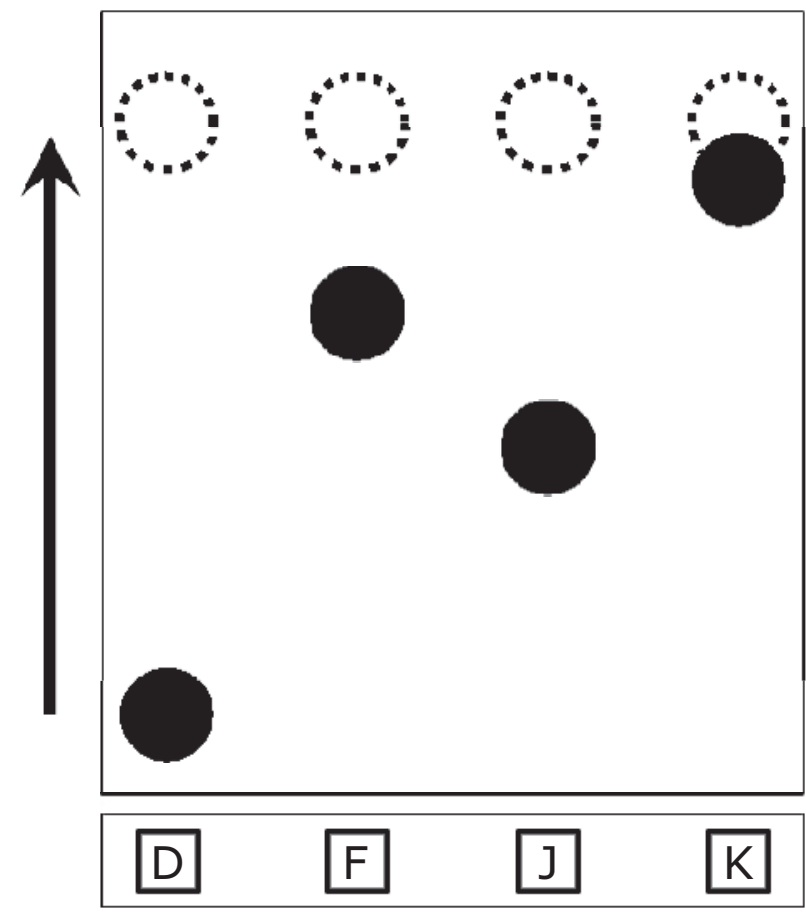

Figure 1. The serial interception sequence learning (SISL) task. Circular cues move vertically upward from the bottom of the screen toward one of four target zones marked as dashed rings. Participants press the corresponding key (D, F, J, or K) on the keyboard and attempt to time their responses so that the key is pressed just as the cue moves through the target zone. As shown, the participant would be timing the press of the " $K$ " key to coincide with the rightmost cue arriving at the vertical circle and immediately planning the subsequent responses " $F$ " then "J" and then "D."

in 60-trial blocks (without noise). The order of the sequences was randomized, and no indication of breaks between the sequences was given. Implicit sequence knowledge was assessed by comparing percentage of correct performance on the practiced sequence with the four foil sequences.

After the implicit knowledge test, participants were informed that there had been a repeating sequence during the initial training phase of the experiment and were given a recognition task. Participants watched and responded to each of the five sequences, each presented twice, and they immediately rated the sequence on a $0-100$ scale as to how likely it was that they had practiced it $(0=$ sure the sequence had not been seen, $50=$ unsure, and $100=$ sure the sequence had been seen). This type of recognition task has been shown to be extremely sensitive to explicit sequence knowledge with the SRT task (Reber \& Squire, 1994, 1998; Willingham et al., 1993). Even fragmentary explicit knowledge of a portion of the sequence is enough to distinguish the trained sequence from the four foils.

The recognition test was followed by a free recall test, in which participants saw the screen with only the yellow targets and used the keyboard to indicate their best guess as to what they thought the repeating sequence had been. Participants were required to generate at least 12 responses. The sequence generated by the participants was compared with all five sequences across all 12 possible starting positions, since the sequence was not necessarily learned with a specific start or end. The longest matching subsequence was identified (the four foils were scored this way to estimate the baseline matching of participants' reports to untrained sequences). The experiment lasted around 45-50 $\mathrm{min}$. 


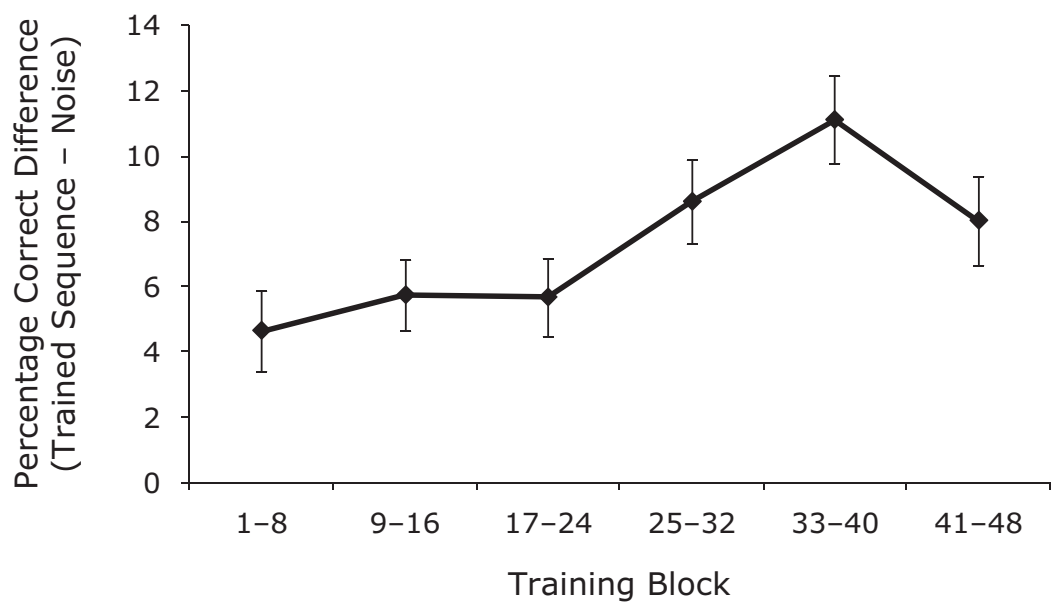

Figure 2. Learning curve throughout training. The learning curve was calculated as the percentage correct difference between the trained sequence blocks and the random noise blocks for groups of eight blocks ( 480 trials). Error bars reflect standard errors of the means.

\section{RESULTS}

During the training portion of the task, which included $20 \%$ pseudorandom noise, participants made an average of $65.01 \%$ correct responses $(S E=1.8 \%)$. Due to the increasing difficulty during training (shortening ISIs), overall task performance remained relatively stable. Performance improvement due to sequence-specific learning was determined by comparing the percentages correct for sequence trials and noise trials for each training block (Figure 2). On the posttraining SISL test with all five sequences, percentage correct performance was examined with a $2 \times 5$ mixed-model ANOVA, with trained sequence versus novel sequences as a within-participants factor and sequence number as a between-participants factor. Participants had reliably better SISL task performance for the trained sequence $(M=60.75 \%, S E=2.49 \%)$ than for the foils ( $M=50.64 \%, S E=1.14 \%)[F(1,25)=29.4, p<$ $.001]$. There was no reliable effect of sequence number, nor any interaction $(F \mathbf{s}<1)$, reflecting no difference in performance or in the learnability of the five sequences.

Recognition test scores were analyzed with a similar $2 \times 5$ mixed-model ANOVA, using trained sequence rating versus mean novel sequences rating as the withinparticipants factor. There was no main effect of trained $(M=50.97, S E=4.36)$ versus foil $(M=47.43, S E=$ 2.51 ) sequences $[F(1,25)=1.09, p>.30]$, indicating no reliable recognition of the repeating sequence. Neither the main effect of assigned sequence nor interaction were reliable $[F(4,25)=2.25, p>.05$, and $F(1,25)=1.57, p>$ .20 , respectively]. The free recall test was scored by comparing the first 12 items reported to the trained sequence and by identifying the length of the longest matching subsequence (longer matches reflecting more accurate memory of the sequence). The participant's report was also scored against each of the other foils to provide an estimate of chance. The recall scores were analyzed with the same mixed-model $2 \times 5$ ANOVA. No reliable effect of target versus foil, sequence, or interaction was found $(F \mathbf{S}<1)$.

To assess individual sequence learning, we compared each participant's percentage correct during SISL performance on the trained sequence with their performance on the foil sequences by using a chi-square analysis (correct vs. incorrect responses for the target sequence and four foils). For 20 of the 30 participants, SISL test performance was reliably greater, with more correct responses made on the trained sequence than on the foil sequences $\left(\chi^{2}>3.84, p<.05\right)$. As can be seen in Figure 3, some participants exhibited high levels of implicit sequence knowledge with virtually no explicit knowledge. ${ }^{1} \mathrm{Al}-$ though there were also individuals who expressed moderate levels of implicit and explicit knowledge, the overall correlation between scores for all participants was $r=$ -.22 (n.s.). Of note, the correlation between implicit and recognition memory scores for just the 20 participants who exhibited individually reliable implicit sequence knowledge was reliably negative $(r=-.60, p<.01)$. Although this correlation was strongly influenced by the participant who scored the worst on the recognition test (but who performed reliably on the implicit test), the correlation without this participant $(r=-.42, p=.07)$ still trended toward significance and is suggestive of possible competition between implicit and explicit memory systems. This data point is also indicative of the variability in the recognition measure. Since a lack of explicit knowledge should lead to an overall recognition score of zero, it is likely that the negative score reflects measurement noise, which suggests that some very positive recognition scores may also reflect measurement noise rather than robust explicit sequence knowledge.

Test performance on the SISL task for participants who scored particularly poorly on both recognition and recall tests (scoring the target sequence lower or identical to foils on both tests ${ }^{2}$ ) was examined to identify learning in participants with no hint of explicit sequence knowl- 


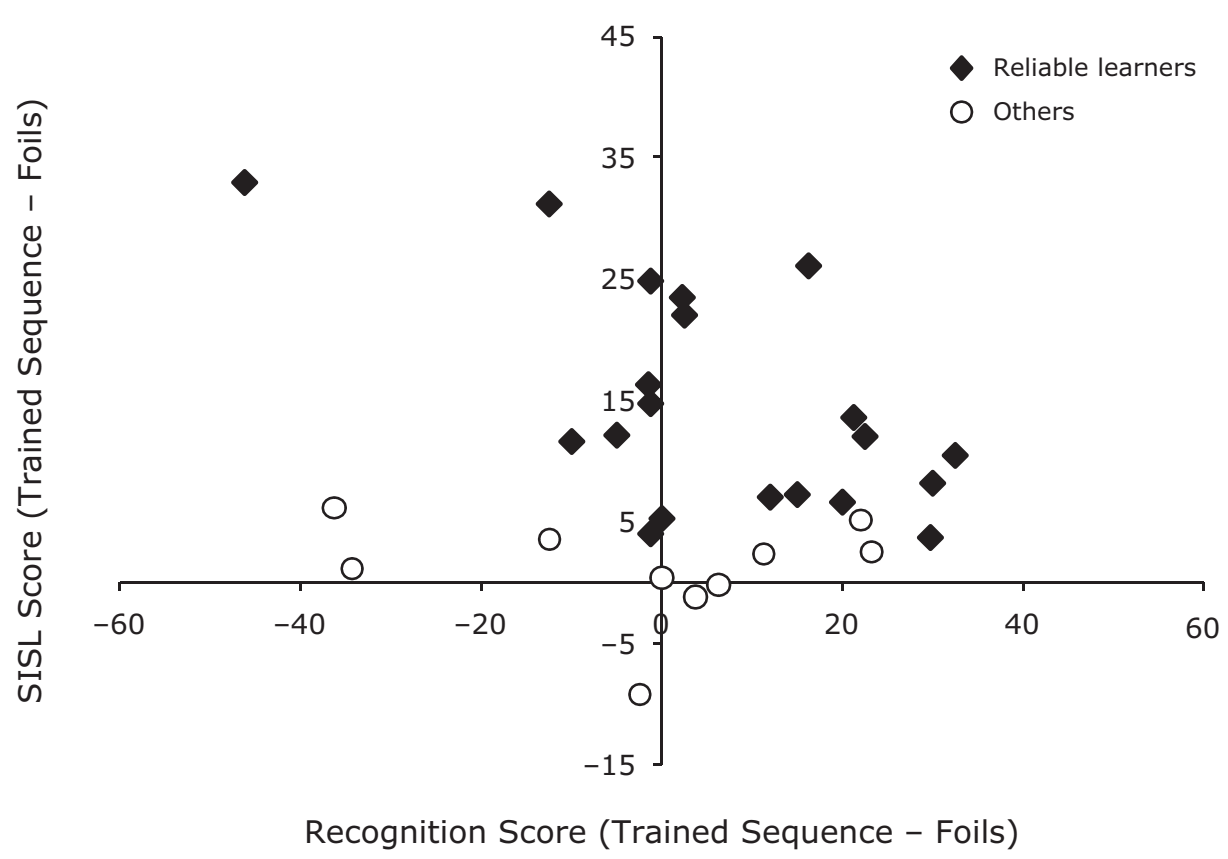

Figure 3. Scatterplot of sequence knowledge assessed implicitly (SISL score) and via recognition. The SISL score is the difference between the mean percentage correct on the trained sequence and the four foils. The recognition score is the difference between the confidence rating for the trained sequence and the average of the four foils. Individuals who were reliable learners (according to the chi-square test) are marked in solid diamonds, whereas those who were not are marked as open circles.

edge. These 11 participants exhibited reliably better SISL test performance for the trained sequence $(M=62.2 \%$, $S E=7.92 \%)$ than for the foil sequences $(M=52.1 \%$, $S E=6.81 \%)[t(10)>2.62, p<.05]$. Their performance did not differ from that of the remaining 19 participants ( $M=59.9 \%, S E=5.43 \%$, for the trained sequence; $M=$ $49.8 \%, S E=5.45 \%$, for the foils). A $2 \times 2$ mixed-model ANOVA of sequence type (trained or foils) and group (with or without any hint of explicit sequence knowledge) revealed the expected effect of sequence $[F(1,28)=29.2$, $p<.001$ ] but showed no main effect of group nor an interaction $(F \mathrm{~s}<1)$. There was no evidence that explicit knowledge led to better implicit test performance.

Although a recognition test is generally sensitive to explicit knowledge, it might be noted that the test of explicit sequence recognition presented each sequence only twice, whereas the implicit test is based on performance data from 20 sequence repetitions. Examining just the first 24 responses ( 2 repetitions) to the trained sequence during the SISL performance test, participants made correct responses on $62.5 \%(S E=5.0 \%)$ of trials compared with an average of $51 \%$ correct responses $(S E=3.95 \%$ ) on the 4 foil sequences [first 24 responses to each foil; $t(29)=3.25$, $p<.01]$. Thus, with exactly the same amount of exposure to the repeating sequence, the implicit test shows a reliable performance increase, whereas participants' attempts to recognize the trained sequence were unsuccessful.

In order to compare the ability of each test to identify the trained sequence, we assessed whether the highest scoring sequence was, in fact, the trained sequence across each of the three tests (SISL performance, recognition, and recall). For the SISL test, in 21 of the 30 participants $(70 \%)$, the trained sequence showed the best performance (by percentage correct responses). For the recognition test, only 4 participants gave the trained sequence their highest recognition rating, 1 rated all five sequences as exactly 50 (tied), and the other 25 all rated at least one of the foils higher than the trained sequence. For the recall test, 4 participants produced a sequence that provided the best match to the trained sequence, 6 participants produced a sequence that matched both the trained sequence and one or more of the foils equally well, and 20 participants produced a sequence that matched one of the foils better than the practiced sequence. For both explicit knowledge tests, discrimination was not appreciably different from chance $(20 \%)$. The ability of each test to identify the trained sequence is shown in Figure 4. The histograms plot the rank of the trained sequence among the foil sequences on the basis of performance for each participant.

\section{DISCUSSION}

Consider a scenario in which, solely on the basis of the test data, one was attempting to deduce the sequence on which a participant had been trained. Choosing the sequence on which the participant made the fewest errors during the implicit test would correctly identify the trained sequence $70 \%$ of the time. In contrast, choosing the sequence to which the participant gave the highest recognition rating or 


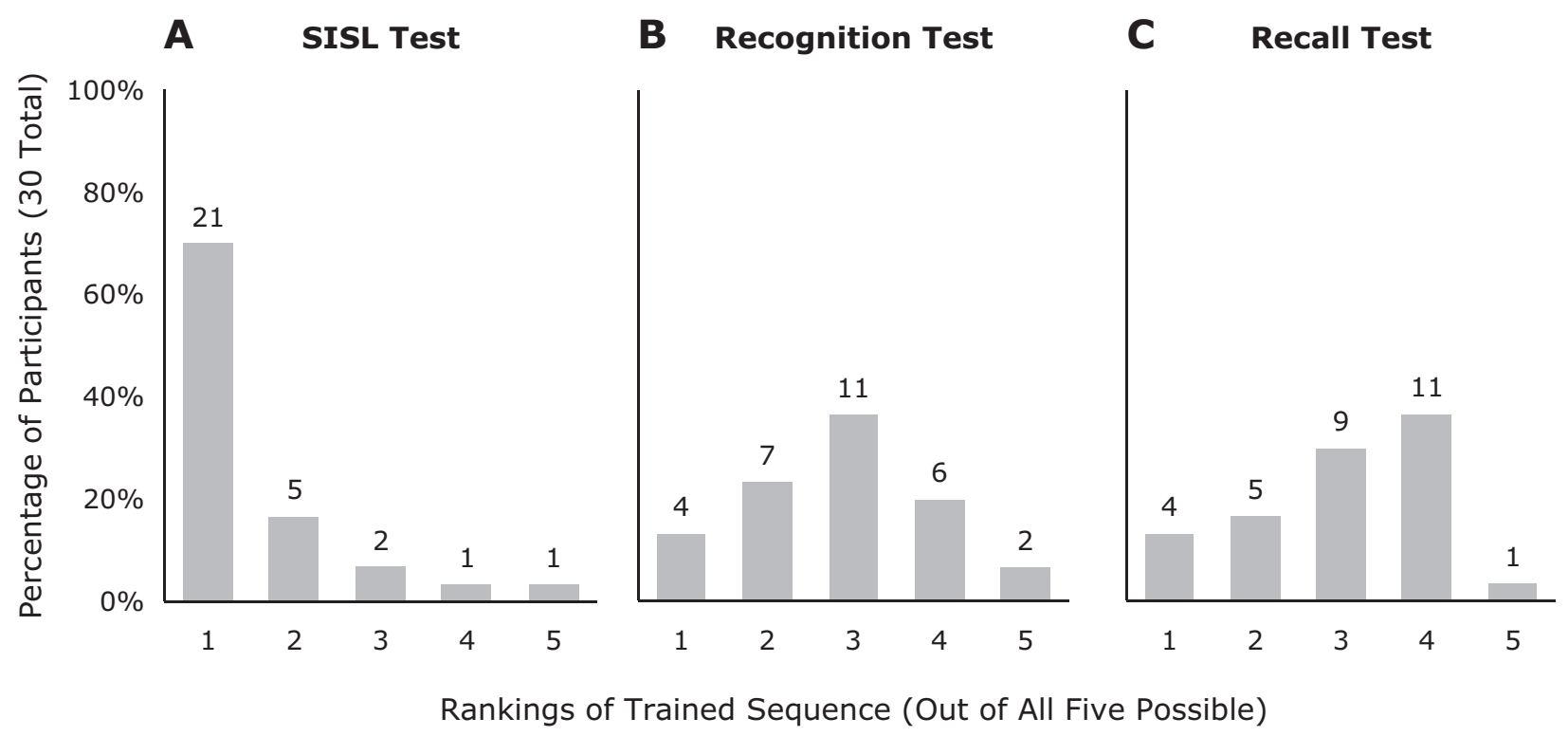

Figure 4. Histograms of trained sequence discriminability for each knowledge test. Numerals at the head of each bar show the number of participants. To assess the ability of each of the three sequence-knowledge tests to discriminate the trained sequence from the foil sequences [implicit: serial interception sequence learning (SISL) performance; explicit: recognition and recall], the scores given to each of the five tested sequences were converted to rankings. The distribution of the rankings of the trained sequence is shown via a histogram for each test. If the trained sequence received the highest score (best performance, highest recognition rating, or best match to the generated recall), it received a ranking of 1 . Tied rankings were scored as the average (e.g., if the trained sequence tied a foil for rankings 3 and 4, it was scored as 3.5). For the histograms, scores were rounded up so that a ranking of 1.5 is shown in the second bin. For the SISL performance test (panel A), 21 participants performed best on the trained sequence. For the two explicit tests, there was virtually no tendency for the trained sequence to receive a better score than the foils. Although 4 participants gave the trained sequence the highest recognition rating (panel B), the recognition ranking given to the trained sequence was very likely to be lower than several of the foils. Likewise, the recall test did not tend to identify the trained sequence (panel C). In terms of the tendency for the tests to correctly pick out the trained sequence, only the SISL performance (implicit) test was effective in discriminating between the trained sequence and the four foils.

best matched their free recall would be dramatically inferior $(13 \%-17 \%$ for both tests). Training on the SISL task leads to memory that can be expressed clearly by motor performance, but not effectively by recognition or recall. This difference in the reliable expression of sequence knowledge in each individual provides an unusually clear dissociation in knowledge representation of implicit and explicit sequence knowledge in healthy participants.

Previous reports of a dissociation between implicit and explicit sequence knowledge in healthy participants have been criticized for the possibility that the explicit tests were insufficiently sensitive to small amounts of knowledge (Shanks \& St. John, 1994; Wilkinson \& Shanks, 2004). Considerable effort has gone into designing tests that would assess explicit knowledge in a more sensitive manner, but some, such as extended free generation of sequences (Smyth \& Shanks, 2008), may not be sensitive to only explicit memory. In contrast, the SISL task produces greatly improved sensitivity to implicit sequence knowledge. In addition to producing individually reliable learning in a number of participants who exhibited no evidence of explicit memory, we can compare the effectiveness of the implicit test and the recognition test in a condition in which the same amount of data is used. Exposure to two repetitions of a repeating sequence is enough to show a reliable difference in performance on the SISL task for the trained sequence, but is insufficient to support an accurate recognition judgment.

Since 12-item sequences are fairly easy for healthy participants to memorize explicitly (e.g., Reber \& Squire, 1998), it is unsurprising that implicit and explicit knowledge often co-occur (Curran \& Keele, 1993; Willingham \& Goedert-Eschmann, 1999; Willingham, Nissen, \& Bullemer, 1989). However, memory systems theory predicts that this co-occurrence is not necessary and that it should be possible for healthy participants to have implicit sequence knowledge without concomitant explicit knowledge, such as the dissociation seen in amnesic patients (e.g., Nissen \& Bullemer, 1987; Reber \& Squire, 1994, 1998). The sequence recognition task used here generally shows evidence of explicit memory in healthy participants (Reber \& Squire, 1994, 1998; Willingham et al., 1993), and, although some participants in the present experiment were able to recognize the sequence they had been practicing, the rate appears to be lower than generally is seen in the SRT task.

A number of variables can contribute to the amount of explicit knowledge developed during sequence learning, and it is likely that several features of the SISL task design contributed to the low levels of explicit knowledge. The SISL task produces constant attentional demands and a high perceptual load, which may assist in producing a strong expression of implicit learning, while simultane- 
ously inhibiting explicit knowledge development (Deroost, Coomans, \& Soetens, 2009). Destrebecqz and Cleeremans (2001) demonstrated a similar reduction in explicit sequence knowledge due to continuous performance demands in the SRT task by reducing the delay between responding and the next cue. In addition, shortening ISIs and interspersing noise trials ( $20 \%$ of trials) throughout the training phase maintains difficulty as participants learn. The fact that the SISL task produces robust implicit knowledge quickly, in spite of the interspersed noise, provides an ideal situation for observing implicit learning without conscious sequence awareness (Fu, Fu, \& Dienes, 2008; Jiménez, 2003).

Learning a motor sequence without developing explicit knowledge probably reflects the roles of the basal ganglia and connected motor cortical structures in sequence learning (Ashe, Lungu, Basford, \& Lu, 2006; Doyon et al., 2009). This learning system is independent of the MTL memory system, which supports declarative memory for facts and events. Damage to the MTL produces impairments in declarative learning, whereas basal ganglia dysfunction produces impairments in motor control, attention-set shifting, and some frontal lobe tests that require management of goals (Grahn, Parkinson, \& Owen, 2008). The results found here reinforce the idea that implicit learning of the practiced sequence is supported primarily by nondeclarative memory via plasticity within corticostriatal circuits (loops connecting motor and premotor areas and the caudate/putamen).

The difference between the explicit knowledge of how to execute an intended motor sequence and expert procedural performance of a sequence is readily clear to anybody who has attempted to teach a skill with a motor component (e.g., teaching a teenager how to drive). However, most naturalistic encounters with this type of learning tend to have both explicit didactic and practice components, making it unclear how separate these systems are. Research on memory systems with healthy participants has shown dissociations between performance and reportability, but it has generally not been possible to rule out a significant contribution of explicit memory or the possibility of a sensitivity difference in knowledge tests (Shanks \& St. John, 1994) when the implicit learning effects are subtle. These problems are overcome in the SISL task by providing a situation in which robust implicit learning can occur in the absence of explicit sequence knowledge. The strong dissociation found here indicates that the implicit learning process can function entirely independently of the explicit system even in healthy participants.

\section{AUTHOR NOTE}

The present work was funded in part by an American Psychological Association Diversity Program in Neuroscience Fellowship to D.J.S. and a Training Grant to support Human Cognitive Neuroscience research (T32-NS047987) to E.W.G. The perceptual-motor task design was inspired by the rhythm games Dance Dance Revolution, developed and published by Konami, and Guitar Hero, developed by Harmonix (published by RedOctane/Activision). Address correspondence to P. J. Reber, Department of Psychology, Northwestern University, 2029 Sheridan Road, Evanston, IL 60208 (e-mail: preber@northwestern.edu).

\section{REFERENCES}

Ashe, J., Lungu, O. V., BASFord, A. T., \& Lu, X. (2006). Cortical control of motor sequences. Current Opinion in Neurobiology, 16, $213-$ 221. doi:10.1016/j.conb.2006.03.008

Brooks, D. N., \& BADDELEY, A. D. (1976). What can amnesic patients learn? Neuropsychologia, 14, 111-122.

Chambaron, S., Ginhac, D., Ferrel-Chapus, C., \& Perruchet, P. (2006). Implicit learning of a repeated segment in continuous tracking: A reappraisal. Quarterly Journal of Experimental Psychology, 59, 845-854. doi:10.1080/17470210500198585

Cohen, N. J., \& SQuire, L. R. (1980). Preserved learning and retention of pattern-analyzing skill in amnesia: Dissociation of knowing how and knowing that. Science, 210, 207-210.

Curran, T., \& Keele, S. W. (1993). Attentional and nonattentional forms of sequence learning. Journal of Experimental Psychology: Learning, Memory, \& Cognition, 19, 189-202.

Deroost, N., Coomans, D., \& Soetens, E. (2009). Perceptual load improves the expression but not learning of relevant sequence information. Experimental Psychology, 56, 84-91.

Destrebecqz, A., \& Cleeremans, A. (2001). Can sequence learning be implicit? New evidence with the process dissociation procedure. Psychonomic Bulletin \& Review, 8, 343-350.

Doyon, J., Bellec, P., Amsel, R., Penhune, V., Monchi, O., CarRIER, J., ET AL. (2009). Contributions of the basal ganglia and functionally related brain structures to motor learning. Behavioural Brain Research, 199, 61-75. doi:10.1016/j.bbr.2008.11.012

FLEGAL, K. E., \& ANDERSON, M. C. (2008). Overthinking skilled motor performance: Or why those who teach can't do. Psychonomic Bulletin \& Review, 15, 927-932. doi: 10.3758/PBR.15.5.927

Fu, Q. F., Fu, X. L., \& Dienes, Z. (2008). Implicit sequence learning and conscious awareness. Consciousness \& Cognition, 17, 185-202.

Gabrieli, J. D. E., Corkin, S., Mickel, S. F., \& Growdon, J. H. (1993). Intact acquisition and long-term retention of mirror-tracing skill in Alzheimer's disease and in global amnesia. Behavioral Neuroscience, 107, 899-910.

Grahn, J. A., Parkinson, J. A., \& Owen, A. M. (2008). The cognitive functions of the caudate nucleus. Progress in Neurobiology, 86, 141-155.

JIMÉNEZ, L. (2003). Intention, attention, and consciousness in probabilistic sequence learning. In L. Jiménez (Ed.), Attention and implicit learning (pp. 43-68). Amsterdam: Benjamins.

Milner, B., Squire, L. R., \& Kandel, E. R. (1998). Cognitive neuroscience and the study of memory. Neuron, 20, 445-468.

Nissen, M. J., \& Bullemer, P. (1987). Attentional requirements of learning: Evidence from performance measures. Cognitive Psychology, 19, 1-32.

Reber, P. J., Gitelman, D. R., Parrish, T. B., \& Mesulam, M. M. (2003). Dissociating explicit and implicit category knowledge with fMRI. Journal of Cognitive Neuroscience, 15, 574-583.

Reber, P. J., \& Squire, L. R. (1994). Parallel brain systems for learning with and without awareness. Learning \& Memory, 1, 217-229.

Reber, P. J., \& Squire, L. R. (1998). Encapsulation of implicit and explicit memory in sequence learning. Journal of Cognitive Neuroscience, 10, 248-263.

Reed, J., \& Johnson, P. (1994). Assessing implicit learning with indirect tests: Determining what is learned about sequence structure. Journal of Experimental Psychology: Learning, Memory, \& Cognition, 20, 585-594.

Schooler, J. W., \& Engstler-Schooler, T. Y. (1990). Verbal overshadowing of visual memories: Some things are better left unsaid. Cognitive Psychology, 22, 36-71.

Shanks, D. R., \& ST. John, M. F. (1994). Characteristics of dissociable human learning systems. Behavioral \& Brain Sciences, 17, 367-447.

Smyth, A. C., \& Shanks, D. R. (2008). Awareness in contextual cuing with extended and concurrent explicit tests. Memory \& Cognition, 36, 403-415.

Squire, L. R., \& Knowlton, B. J. (2000). The medial temporal lobe, the hippocampus, and the memory systems of the brain. In M. S. Gazzaniga (Ed.), The new cognitive neurosciences (pp. 765-776). Cambridge, MA: MIT Press.

WiLKINSON, L., \& SHANKS, D. R. (2004). Intentional control and im- 
plicit sequence learning. Journal of Experimental Psychology: Learning, Memory, \& Cognition, 30, 354-369.

Willingham, D. B., \& Goedert-EschmanN, K. (1999). The relation between implicit and explicit learning: Evidence for parallel development. Psychological Science, 10, 531-534.

Willingham, D. B., Greeley, T., \& Bardone, A. M. (1993). Dissociation in a serial response time task using a recognition measure: Comment on Perruchet and Amorim (1992). Journal of Experimental Psychology: Learning, Memory, \& Cognition, 19, 1424-1430.

Willingham, D. B., Nissen, M. J., \& Bullemer, P. (1989). On the development of procedural knowledge. Journal of Experimental Psychology: Learning, Memory, \& Cognition, 15, 1047-1060.

Willingham, D. B., Salidis, J., \& Gabrieli, J. D. E. (2002). Direct comparison of neural systems mediating conscious and unconscious skill learning. Journal of Neurophysiology, 88, 1451-1460.

\section{NOTES}

1. As can be seen in Figure 3, there are instances in which reliable learners have a lower SISL score than do those who were not statistically reliable according to the chi-square test. This is due to the positive skew of the $\chi^{2}$ distribution. For example, a participant who achieved $99 \%$ correct on the trained sequence and $96 \%$ correct on the foil sequence had a significant effect of learning. However, a participant who achieved $51 \%$ and $45 \%$ correct on the trained and foil sequences, respectively, would not show significant learning.

2. Note that this criterion for lack of explicit knowledge is particularly conservative. A group of participants who have no explicit knowledge should be expected to exhibit a mean score of zero on recognition or recall tests. However, test noise means that individual scores should distribute normally around zero - half greater than zero, and half less than zero. By restricting the analysis to those who scored zero or less on both tests, it is likely that we are excluding a number of participants who have essentially no explicit knowledge but happened to score just above zero on either recognition or recall.

(Manuscript received February 18, 2010; revision accepted for publication May 21, 2010.) 